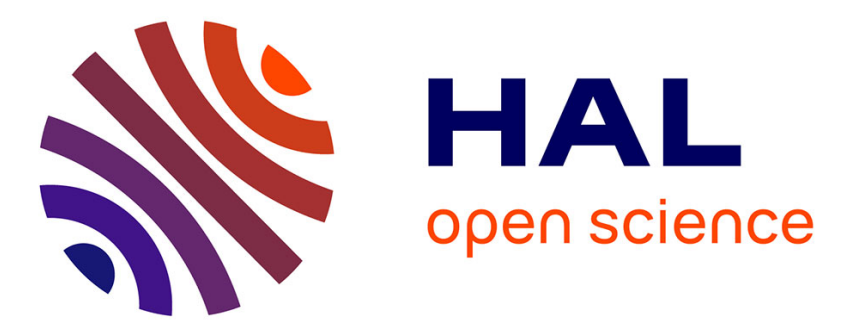

\title{
Broadband Passive Two-Feed-Per-Beam Pillbox Architecture for High Beam Crossover Level
}

Thomas Potelon, Mauro Ettorre, Terry Bateman, Jim Francey, Ronan Sauleau

\section{To cite this version:}

Thomas Potelon, Mauro Ettorre, Terry Bateman, Jim Francey, Ronan Sauleau. Broadband Passive Two-Feed-Per-Beam Pillbox Architecture for High Beam Crossover Level. IEEE Transactions on Antennas and Propagation, 2020, 68 (1), pp.575-580. 10.1109/TAP.2019.2934353 . hal-02469489

HAL Id: hal-02469489

https://hal-univ-rennes1.archives-ouvertes.fr/hal-02469489

Submitted on 20 Mar 2020

HAL is a multi-disciplinary open access archive for the deposit and dissemination of scientific research documents, whether they are published or not. The documents may come from teaching and research institutions in France or abroad, or from public or private research centers.
L'archive ouverte pluridisciplinaire HAL, est destinée au dépôt et à la diffusion de documents scientifiques de niveau recherche, publiés ou non, émanant des établissements d'enseignement et de recherche français ou étrangers, des laboratoires publics ou privés. 


\title{
Broad-Band Passive Two-Feeds-Per-Beam Pillbox Architecture for High Beam Cross-Over Level
}

\author{
Thomas Potelon, Mauro Ettorre, Senior Member, IEEE, Terry Bateman, Jim Francey and Ronan \\ Sauleau, Fellow, IEEE
}

\begin{abstract}
Multi-beam antennas based on quasi-optical systems feeding a single radiating aperture generate orthogonal beams with a low crossover level between adjacent beams (around $-13 \mathrm{~dB}$ at most). To circumvent this limitation, we present a circuit architecture allowing the excitation of a quasi-optical system (pillbox system) with two feeds per beam to reach much higher beam cross-over levels. To this end, a specific 8-beam passive circuit is designed to cover the $\mathbf{7 6 - 8 6 ~ G H z}$ frequency band. It is based on a single-layer substrate integrated waveguide (SIW) coupler followed by equi-phase SIW lines. The maximum phase and amplitude imbalance between the sources are only $35^{\circ}\left(\lambda_{g} / 10\right.$, with $\lambda_{g}$ the wavelength in the SIW lines at the design frequency) and $1.3 \mathrm{~dB}$ respectively, and the isolation is better than $-19 \mathrm{~dB}$. The measured beam cross-over level of the corresponding antenna is better than $-3.2 \mathrm{~dB}$, corresponding to an improvement of $16 \mathrm{~dB}$ with respect to single-feed-per-beam pillbox systems. The proposed passive architecture offers beam cross-over levels suitable for low-cost electronically controlled multi-beam applications, as for next-generation $5 \mathrm{G}$ backhauling systems.
\end{abstract}

Index Terms - Cross-over level, multi-beam antennas, multiple-feeds-per-beam, pillbox, SIW technology.

\section{INTRODUCTION}

Electronically-controlled multi-beam antennas are required in many high-gain applications such as radar systems, satellite communications, or next-generation cellular networks, etc. Phased arrays [1] are the most agile solution, and yet for high gain applications at millimeter-wave frequencies (automotive radars, 5G fronthaul and backhaul antennas, etc.) the required large number of phase shifters make them complex and lossy. On the other hand, passive beam formers are usually more efficient and easier to fabricate at a lower cost but with limited performance in terms of scanning [2]. In this paper, we investigate an innovative multiple-feed-per-beam architecture made of a continuous transverse stub (CTS) antenna fed by a pillbox system [3]. The system is designed in E-band (71-86 $\mathrm{GHz}$ ). The antenna was originally described with a single fixed beam in [4], and its architecture is briefly recalled in Section II.A.

The pillbox coupler is a beam former based on a 2D parabolic reflector integrated in a parallel plate waveguide (PPW) environment. Beam scanning can be easily obtained by simply displacing the feeding source in the focal plane of the parabolic reflector.

The maximum cross-over level between consecutive beams achievable with passive beam formers (either circuit-based or quasioptical systems) is subject to physical limitations [2], [5], [6]. More specifically, [7] shows that classical pillbox antennas with one-feedper-beam (1FPB) achieve a cross-over level of around -19 dB with negligible losses. This level cannot be improved without encountering a significant rise of the edge tapering, thus leading to a higher side lobe

This work was supported by the European Union Seventh Framework Programme (FP7/2007-2013) through MiWaveS project grant 619563.

This work is also supported by the European Union through the European Regional Development Fund (ERDF), and by Ministry of Higher Education and Research, Brittany and Rennes Métropole, through CPER Project SOPHIE/STIC \& Ondes. level (SLL), important spillover loss, and significant degradation of the antenna efficiency. More precisely, we show here that a cross-over level between adjacent beams higher than $-13 \mathrm{~dB}$ cannot be produced without a rise of the spillover loss to more than $1.5 \mathrm{~dB}$ (see Section III). To circumvent this limitation, a practical solution consists in adopting two separate radiating apertures generating two interleaved sets of beams, as reported in [7] and [8]. This solution provides a beam cross-over level in the $-3 \mathrm{~dB}$ range with clean patterns, but at the cost of a reduced aperture efficiency and increased footprint of the antenna system. Another simpler solution to improve the beam cross-over level consists in using multiple focal feeds per beam as in [9] for classical parabolic reflectors.

In this paper, we investigate the use of overlapping focal sources for a non-tapered two-feed-per-beam (2FPB) excitation for high crossover between consecutive beams. This approach differs significantly from [9] since the latter uses a tapered power repartition among the feeds, resulting in a cross-over level limitation. The beam overlapping is achieved by designing a novel beam interlacing circuit (BIC) based on passive couplers in SIW technology. The concept is applied to an 8-beam antennas at E-band with a high cross-over level.

To the best of our knowledge, this is the first time that a passive circuit achieves a non-tapered multiple-feed-per-beam excitation with less than $3 \mathrm{~dB}$ loss. This enables to realize a high beam cross-over level with a single radiating aperture.

The paper is organized as follows. In Section II, the antenna architecture is presented for single- and multi-feed-per-beam solutions. Section III explains why a multiple-feed-per-beam excitation is needed, while Section IV presents the BIC design allowing high crossover levels. In section $\mathrm{V}$, the fabrication, simulation and measurement results are provided. Finally, conclusions are drawn in Section VI.

\section{ANTENNA ARCHITECTURE}

\section{A. Single-feed-per-beam antenna}

The single-feed-per-beam antenna was originally described in [4]. It is illustrated in Fig. 1. It comprises 32 radiating slots consisting of vertically-oriented truncated PPWs supporting a quasi-TEM mode. This CTS array is fed by a PPW-based corporate feeding network in metal-only technology. This feeding scheme shapes the beam in Eplane. The pillbox system, placed horizontally underneath the corporate network, generates the line-source excitation needed to feed the corporate feeding network and shapes the main beam in H-plane. It is realized on a two-layer PCB board $(0.508 \mathrm{~mm}$-thick Rogers 3003 , with $\varepsilon_{r}=3.08$ and $\tan (\delta)=0.0010$ at $78.5 \mathrm{GHz}$ ). The pillbox parabolic reflector and focal horn are realized in SIW technology. A multi-slot coupler is implemented to connect the input and output PPWs. The diameter $D$ and focal length $F$ of the parabolic reflector equal $80 \mathrm{~mm}$ and $50 \mathrm{~mm}$, respectively. A specific PCB/Air transition

T. Potelon, M. Ettorre and R. Sauleau are with Univ Rennes, CNRS, Institut d'Electronique et de Télécommunications de Rennes (IETR) - UMR 6164, F35000 Rennes, France (e-mail: thomas.potelon@univ-rennes1.fr).

T. Bateman and J. Francey are with Optiprint AG, Auerstrasse 37, CH-9442 Berneck, Switzerland (e-mail: t.bateman@optiprint.co.uk, j.francey@optiprint.co.uk). 
is used to combine the two modules. The antenna is fed by a standard WR12 waveguide through a WR12-to-SIW transition. Measurements showed that the antenna covers the $71-86 \mathrm{GHz}$ band with a reflection coefficient lower than $-13.5 \mathrm{~dB}$ and a realized gain higher than 29.3 $\mathrm{dBi}$. The measured peak gain was $30.8 \mathrm{dBi}$ at $82.25 \mathrm{GHz}$.

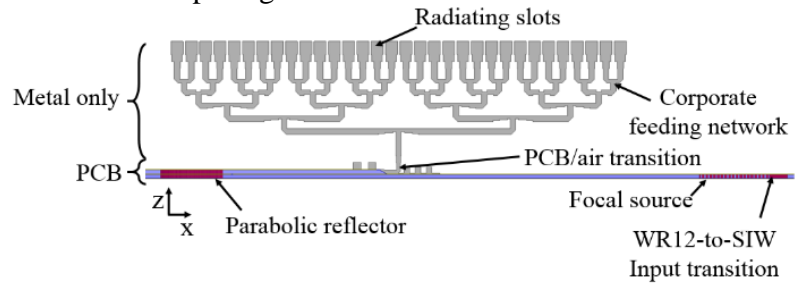

(a)

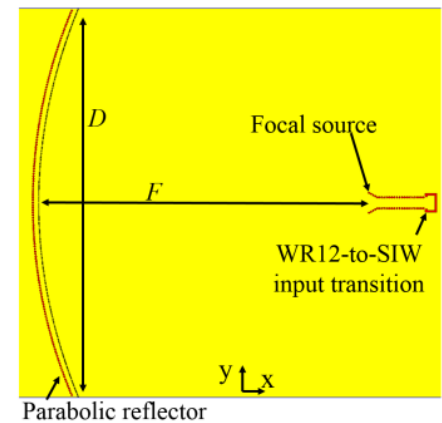

(b)

Fig. 1: Single-feed-per-beam antenna [4]: cross-section (a) and bottom (b) views.

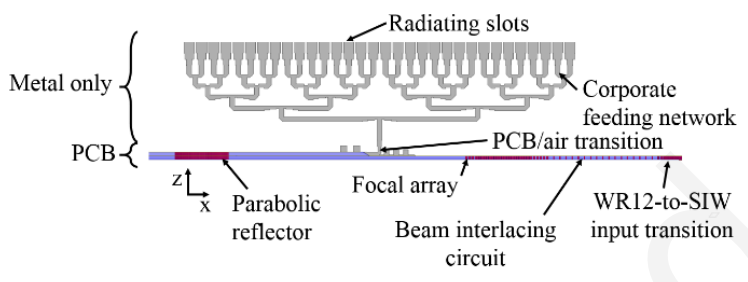

(a)

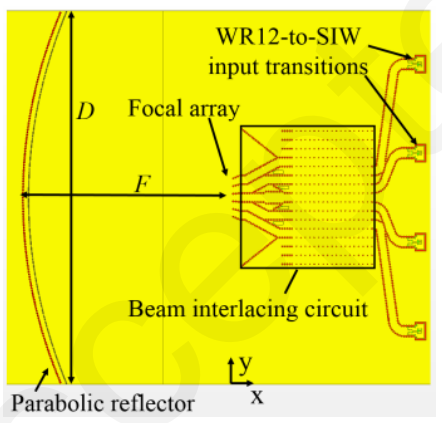

(b)

Fig. 2: Multi-feed-per-beam antenna: cross-section (a) and bottom (b) views.

\section{B. Multi-feed-per-beam antenna}

An overview of the antenna presented in this paper is shown in Fig. 2 . The slot array, the corporate feeding network, the PCB-air transition, the pillbox system and the input transitions are identical to those described in [4]. The main innovation relies on the new BIC and the pillbox focal array, both fabricated in PCB technology. It is worth picturing the antenna as the cascade of three independent functional parts:

- The pillbox system excited by the BIC is responsible for the radiation pattern in $\mathrm{H}$-plane (i.e. $y z$ plane),
- The corporate feeding network controls the radiation pattern in Eplane (i.e. $x z$ plane),

- The slot array radiates in free space the mode guided by the corporate feeding network.

This implies that the radiation patterns along the E-plane of the singleand multi-feed-per-beam antennas are identical.

\section{Multi-BEAM PILLBOX}

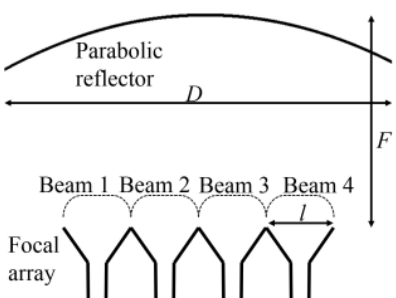

(a)

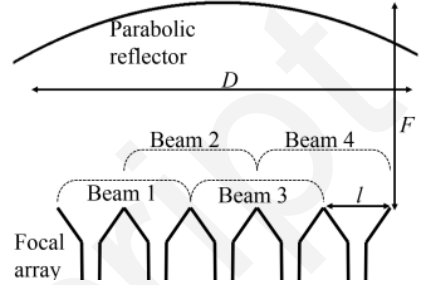

(b)
Fig. 3: Pillbox system and equivalent feeding aperture for each beam (schematic for a 4-beam antenna): (a) one-feed-per-beam (1FPB) excitation; (b) two-feed-per-beam (2FPB) excitation (in this case, the same feed horn is shared by two beams).

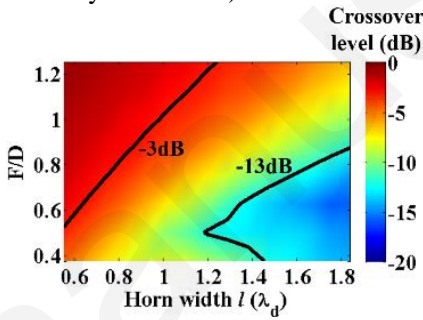

(a)

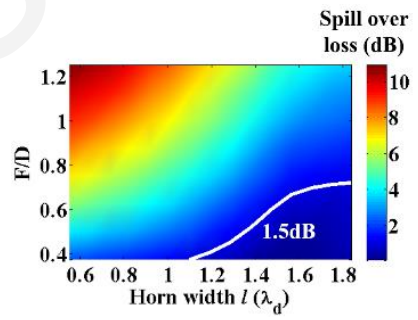

(b)
Fig. 4: Beam crossover level (a) and spill over loss (b) as a function of the $F / D$ ratio and the horn width for the 1 FPB excitation.

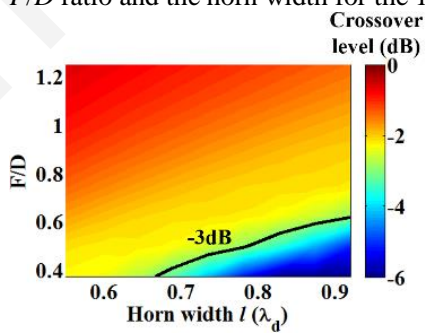

(a)

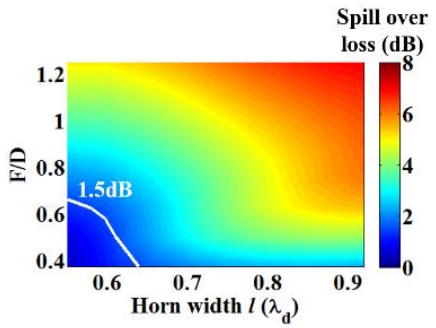

(b)
Fig. 5: Beam crossover level (a) and spill over loss (b) as a function of the $F / D$ ratio and the horn width for the $2 \mathrm{FPB}$ excitation.

In multi-beam pillbox designs, a trade-off must be found between the spillover loss and the beam cross-over level. To do so, the design variables are the parabola diameter $D$, the focal length $F$ and the horn aperture width $l$, as depicted in Fig. 3 (a).

The cross-over level depends on the angular separation between the beams, i.e. the distance between the phase centers of the focal sources. For a fixed value of $F$, the closer the sources, the higher the cross-over level. On the other hand, the spillover loss depends on the illumination of the parabolic reflector by the focal sources. For a fixed $F / D$ ratio, the wider the sources, the lower the spillover is. However, the sources being arranged side by side, the width of the focal sources is limited by the periodicity of the focal array. Therefore small focal sources allows a close arrangement, resulting in a high cross-over level, at the expense of important spillover loss, and inversely, wide sources lead to reduced spillover loss at the expense of a low cross-over level because of the large distance between the sources.

A Geometrical Optics (GO) tool was implemented to define the best trade-off between the various parameters providing a cross-over level higher than $-3 \mathrm{~dB}$ with less than $1.5 \mathrm{~dB}$ spill-over loss. The results are shown in Figs. 4 and 5 at $78.5 \mathrm{GHz}$ as a function of the focal length to parabola diameter ratio $F / D$ and the focal source width $l$. 
The results obtained for the classical 1FPB architecture (Fig. 3 (a)) are represented in Fig. 4. A cross-over level better than $-13 \mathrm{~dB}$ cannot be obtained without significant spillover loss (beyond $1.5 \mathrm{~dB}$ ).

Using a 2FPB excitation allows to enhance the directivity of the focal sources while keeping the same distance between them. Each source is shared between two adjacent beams, as schematized in Fig. 3 (b). The results using this configuration are shown in Fig. 5. A crossover level better than $-3 \mathrm{~dB}$ can be obtained for any source width $l$ with a $F / D$ ratio higher than 0.6. However, the spillover loss is higher for wider sources due to the appearance of grating lobes. For this reason, the sources are designed with the smallest width $\left(0.6 \times \lambda_{d}\right)$ to guaranty a proper $\mathrm{TE}_{10}$ mode propagation over the full $71-86 \mathrm{GHz}$ bandwidth. The $F / D$ ratio is then set to 0.625 as a trade-off between the spillover loss and cross-over level. Note that in order to insure a proper compatibility between the building blocks, the parabola diameter $D$ has to match the radiating slot length $(80) \mathrm{mm}$, thus leading to a focal length of $50 \mathrm{~mm}$. This $2 \mathrm{FPB}$ configuration is expected to provide a -2 $\mathrm{dB}$ crossover level with only $1.5-\mathrm{dB}$ spillover loss. This is extremely advantageous as compared to the $-13 \mathrm{~dB}$ cross-over with identical spillover loss provided by the $1 \mathrm{FPB}$ configuration.

\section{BIC DESIGN}

\section{A. BIC Architecture}

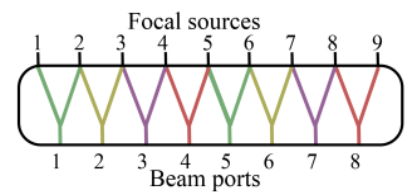

Fig. 6: Schematic view of the BIC.

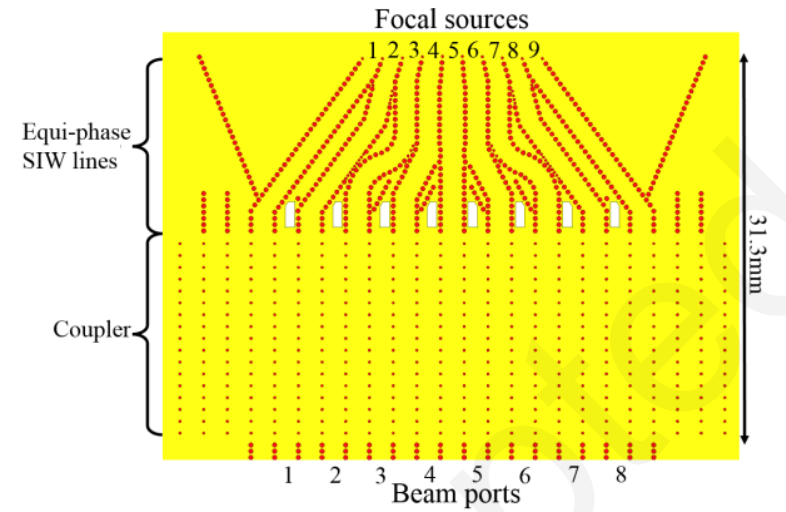

Fig. 7: Designed 8-beam BIC.

A BIC is needed to obtain the 2FPB excitation, as schematized in Fig. 6. It comprises 8 input ports (Beam ports), corresponding to the 8 radiated beams, and 9 outputs ports connected to the 9 focal sources. To guarantee a proper excitation of the beams, two conditions must be satisfied:

- The two active outputs must be excited with the same amplitude and phase,

- The inactive input and output ports must be well isolated (below -15dB) to avoid interferences.

However obtaining this behavior with a lossless passive system is not feasible [5], [6]. For a reduced antenna complexity, active components are avoided and a passive but slightly lossy solution is thus preferred here.

The BIC developed here (Fig. 7) is realized on a single substrate with standard SIW technology (0.508 mm-thick Rogers 3003, with $\varepsilon_{r}=3.08$ and $\tan (\delta)=0.0010$ at $78.5 \mathrm{GHz}$ ), and is composed of a coupler followed by equi-phase SIW lines. Dummy ports are placed at the terminations of the unused coupler outputs. This simple solution provides a true non-tapered 2FPB excitation for reduced beam cross- over level with less than $2 \mathrm{~dB}$ theoretical losses, as shown in the following. For comparison, in [10] the authors proposed a network architecture based on power dividers and $3 \mathrm{~dB}$ couplers that provides $3 \mathrm{~dB}$ theoretical loss in the resistive terminations of the couplers.

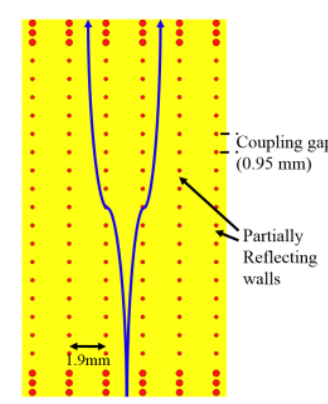

(a)

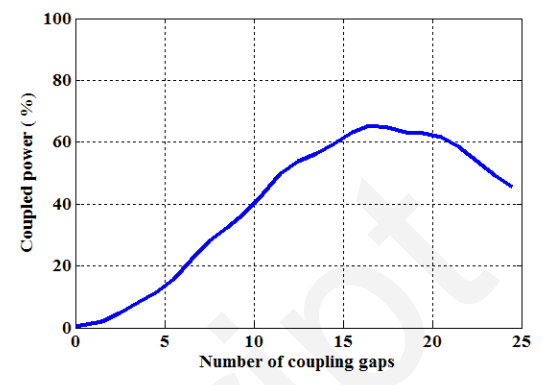

(b)
Fig. 8: SIW coupler (a) and power coupled to the two adjacent waveguides as a function of the number of coupling gaps (b).

\section{B. Coupler}

The SIW coupler designed here is presented in Fig. 8 (a). Adjacent waveguides are separated by metallized via holes behaving like partially reflecting walls. In this way, the $\mathrm{TE}_{10}$ mode coming from the input waveguide couples energy to the adjacent waveguides while propagating. The operation mechanism is similar to the one described in [11], and a very accurate analytical model is provided in the appendix. The symmetry of the structure implies that feeding the coupler by one input will excite the two adjacent waveguides with exactly the same phase and amplitude. Therefore, the beam overlapping function can be achieved by feeding one out of every two waveguides, as depicted in Fig. 7.

The waveguides are sized as wide as possible $(1.9 \mathrm{~mm})$ to shorten the wavelength of the $\mathrm{TE}_{10}$ mode, in order to reduce the overall size of the system. Likewise, using wide gaps between the vias $(0.95 \mathrm{~mm})$ improves the coupling, as well as using small vias $(0.1 \mathrm{~mm}$ in diameter). The coupled power can be controlled by tuning the number of coupling gaps, as shown in Fig. 8 (b). The coupler designed here includes 18 coupling gaps. This configuration provides the highest power coupled to the adjacent waveguides ( $65 \%$ of the input power). The rest of the power either remains in the input waveguide, or couples to more external waveguides.

\section{Equi-phase SIW lines and focal sources}

The focal sources are realized by open-ended SIW lines of $1.3 \mathrm{~mm}$ width (see Fig. 7) and the vias have a diameter of $0.35 \mathrm{~mm}$ with a pitch of $0.5 \mathrm{~mm}$. These sources are connected to the coupler outputs through SIW lines, as depicted in Fig. 7 and Fig. 9 (a). Since the sources are used in pairs, they need to be fed in-phase. Therefore the SIW-lines must be equi-phase in order to preserve the phase uniformity between the coupler outputs. Unfortunately, a slight difference between their physical lengths is unavoidable to arrange them in a compact fashion: they range from $16 \mathrm{~mm}$ for the external SIW lines to $13 \mathrm{~mm}$ for the central one (see Fig. 9 (a)). The phase-shift created by the length differences is lower than $110^{\circ}$, and it is easily compensated by modifying the width of the lines (from $1.6 \mathrm{~mm}$ for the external lines to $2 \mathrm{~mm}$ for the central one).

A phase difference lower than $35^{\circ}$ (corresponding to $\frac{\lambda_{g}}{10}$ with $\lambda_{g}=$ $2.5 \mathrm{~mm}$ at $78.5 \mathrm{GHz}$ ) is obtained from 76 to $86 \mathrm{GHz}$ between two adjacent lines. This guarantees the in-phase excitation of the two sideby-side active sources, thus illuminating the parabolic reflector with a clean and symmetrically tapered power distribution. 


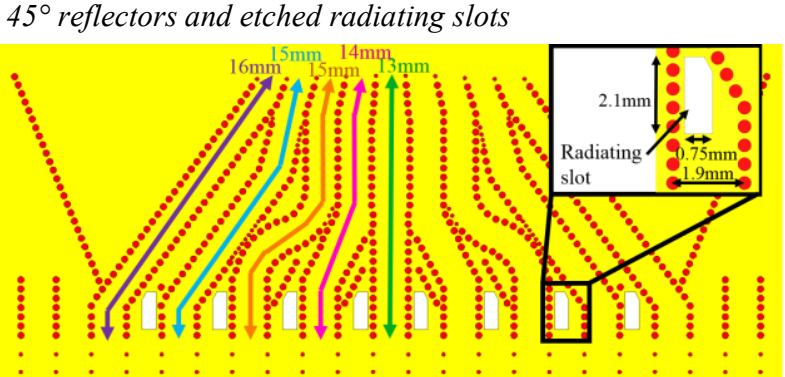

Fig. 9: Equi-phase SIW lines and dummy port realized by a radiating slot.

As it can be seen in Fig. 8, 35\% of the input accepted power is not properly coupled to the adjacent waveguides. This power must be evacuated to avoid internal reflections which would result in appearance of standing waves, thus altering the proper operation of the entire system. The very simple solution proposed here consists in radiating this unwanted power toward the edges or the backside of the antenna. Even though this has very little effect on the radiation patterns, the use of resistive materials may be an alternative option to prevent from back-radiation for sensitive applications.

$45^{\circ}$ reflector walls are placed on both sides of the SIW lines to redirect the unwanted power towards the open-ended edges of the $\mathrm{PCB}$, where it is then radiated into free space. Since such a solution is not applicable to the waveguides embedded between the SIW lines, radiating slots are used. A single radiating slot placed at the tapered short-circuit termination of the waveguides (Fig. 9 (b)) is sufficient to keep the reflection coefficient below $-15 \mathrm{~dB}$ over the full bandwidth.

\section{ANTENNA PERFORMANCES}

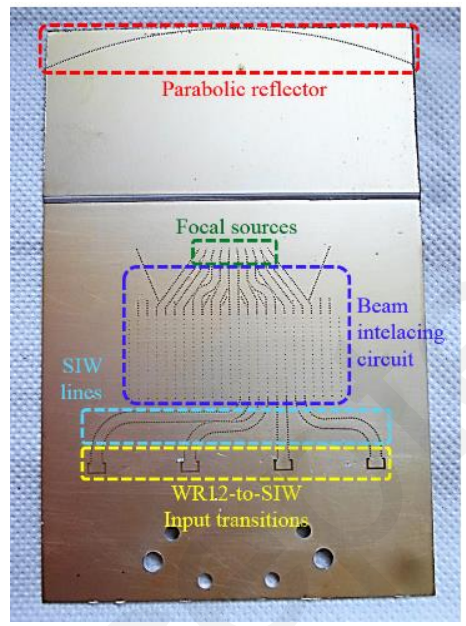

(a)

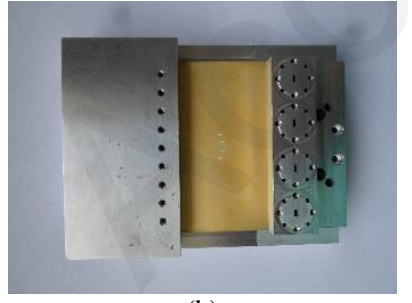

(b)

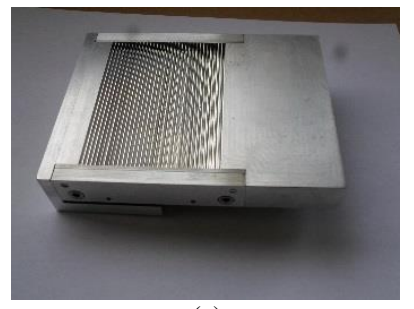

(c)
Fig. 10: Fabricated prototype: PCB of the antenna with 2FPB excitation (a), rear-view of the entire antenna module (b) and top-view of the antenna module (c).

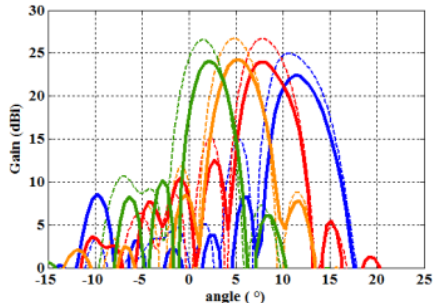

(a)

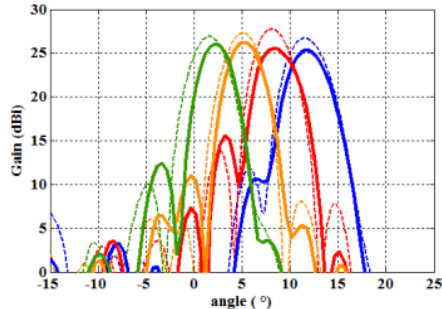

(c)

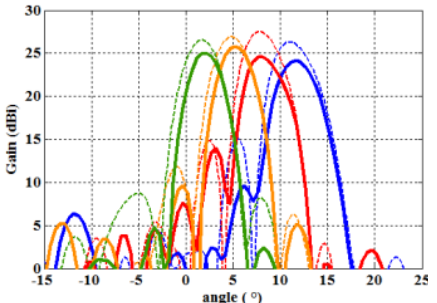

(b)

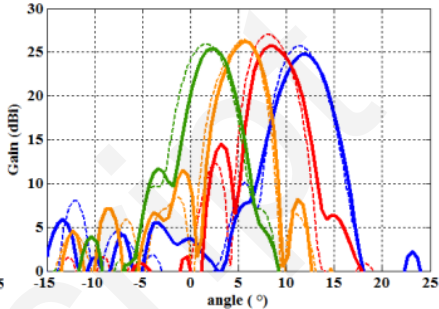

(d)
Fig. 11: Measured (solid curves) and simulated (dotted curves) radiation patterns in H-plane using the 8-beam BIC: at $76 \mathrm{GHz}$ (a), $79 \mathrm{GHz}$ (b), 82 $\mathrm{GHz}(\mathrm{c})$ and $86 \mathrm{GHz}(\mathrm{d})$.

The final PCB module is presented in Fig. 10 (a). Only 4 WR12-toSIW input transitions, identical to the one presented in [4], were implemented for space constraints. These input ports corresponds to 4 different beams pointing at positive elevation angles. However, the symmetry of the entire antenna guarantees similar performance for the 4 remaining beams (negative elevation angles). The input transitions are connected to the BIC inputs by means of SIW lines of various length (from $11 \mathrm{~mm}$ to $36 \mathrm{~mm}$ ), causing losses between $0.4 \mathrm{~dB}$ and 1.5 $\mathrm{dB}$, respectively. The antenna was fabricated and assembled using the PCB-air hybrid technology described in [4]. The slot array and corporate feed network are made by milling aluminum pieces that are assembled using screws and pins. This process guarantees a fabrication accuracy and an assembly alignment both in the $10 \mu \mathrm{m}$ range. The PCB board containing the pillbox system and the BIC is placed underneath, and a specific transition is used to make the two blocks compatible: quarter-wavelength chokes are used to avoid leakage in case of misalignment between the different antenna pieces. More details can be found in [4].

The prototype (Fig. 11 (b) and (c)) was characterized experimentally at IETR with a compact antenna test range, and the simulation results presented for comparison in Fig. 11 were obtained with Ansys HFSS version 19.2. Good agreement is obtained from $76 \mathrm{GHz}$ to $86 \mathrm{GHz}$ and for all the beams. The difference in gain between simulations and measurements is in the range of $0.2-2.7 \mathrm{~dB}$ in the operating band. This is attributed to fabrication inaccuracies and assembly misalignments. In details, inaccuracies in the $30 \mu \mathrm{m}$ range at the equi-phase SIW lines level result in about $70^{\circ}$ phase difference between adjacent focal sources. As a result, the parabolic reflector illumination is deteriorated, the antenna directivity is lowered and the spillover loss increases. The gain is stable over the frequency band and among the beams varying from $22.3 \mathrm{dBi}$ to $25.7 \mathrm{dBi}$. The different lengths of the feeding lines are partly responsible for the gain difference among the beams. The antenna provides an angular coverage of $\pm 13^{\circ}$ with 8 beams, and the beam cross-over is less than $3.2 \mathrm{~dB}$. The SLL remains below $-10 \mathrm{~dB}$ and the cross-polarization level is lower than $-35 \mathrm{~dB}$. Table I compares the proposed 2FPB antenna performance with state-of-the-art millimeter-wave antennas. For a fair comparison, these results do not take into account the losses entailed by feeding features (long input lines and active beam switching components). It emerges that the proposed antenna system produces one of the lowest beam cross-over level. The antenna presented in [8] achieves a similar cross-over level but at the expense of a very large system with only about $41 \%$ aperture 
TABLE I: COMPARISON WITH STATE-OF-THE-ART MILLIMETER-WAVE MULTI-BEAM ANTENNAS

\begin{tabular}{|c|c|c|c|c|c|c|}
\hline Ref. & Antenna type & $\begin{array}{c}\text { Freq. } \\
\text { band } \\
(\mathrm{GHz})\end{array}$ & $\begin{array}{c}\text { Peak } \\
\text { gain } \\
(\mathbf{d B i}) \\
\end{array}$ & $\begin{array}{l}\text { Beam cross- } \\
\text { over (dB) }\end{array}$ & $\begin{array}{c}\begin{array}{c}\text { Aperture } \\
\text { efficiency }\end{array} \\
(\%)\end{array}$ & Size $\left.\left(\lambda_{0}\right)^{3}\right)$ \\
\hline [12] & Rotman lens + patch array & $57-65$ & n.a & -4 & n.a & $6 \times 5 \times 0.06$ \\
\hline [13] & Multi-reflector pillbox + open-ended PPW & 186 & 36.5 & -13.5 & n.a. & $\begin{array}{c}400 \times 400 \times \\
0.3\end{array}$ \\
\hline$[14]$ & Butler matrix + slot array & $28-31$ & 16 & -6 & n.a & $4 \times 1.2 \times 0.5$ \\
\hline$[8]$ & Dual-pillbox system + CTS & $57-66$ & 14.7 & -3 & $41 \%$ & $20 \times 10 \times 0.6$ \\
\hline This work & Pillbox system + CTS array (2FPB) & $76-86$ & 25.7 & -3.2 & $67 \%$ & $43 \times 25 \times 9$ \\
\hline
\end{tabular}

efficiency. On the other hand, the antenna architecture introduced here has an aperture efficiency close to $70 \%$ since the same radiating aperture is used for all beams.

The pillbox excitation has no effect on the E-plane radiation pattern, only driven by the corporate feeding network and the slot array. The half-power beamwidth in E-plane varies from $3.5^{\circ}$ at $(76 \mathrm{GHz})$ to $2.9^{\circ}$ at $(86 \mathrm{GHz})$ and the SLL is in the $-13 \mathrm{~dB}$ range [4].

\section{CONCLUSIONS}

We designed a multi-feed-per-beam circuit in PCB technology to illuminate the pillbox coupler of a multi-beam CTS array. Multi-feedper-beam systems, in contrast to single-feed-per-beam counterparts, are shown to provide high cross-over level between consecutive beams, thus offering an enhanced radio coverage. An 8-beam antenna using two-feeds-per-beam approach was designed, fabricated and characterized over the $76-86 \mathrm{GHz}$ band. Experimental results validated the concept showing an angular coverage of $\pm 13^{\circ}$ with a beam crossover of $-3.2 \mathrm{~dB}$. This corresponds to a $16 \mathrm{~dB}$ improvement compared to a classic 1FPB pillbox-based antenna [7]. The total losses of the BIC are about $3 \mathrm{~dB}$; and according to simulations, using low-loss materials or metal-only technologies could reduce the BIC overall loss to only $1.9 \mathrm{~dB}$.

The proposed architecture can be used with integrated quasi-optical systems. It is key-enabler for single aperture electronically switchable antennas with a high beam cross-over level. This system is suitable for E-band $5 \mathrm{G}$ backhauling applications in which multiple beam coverage is necessary to insure a proper antenna alignment at any time, even in case of inaccurate positioning or disturbing weather conditions. It is also a relevant solution for radar systems and on-board antennas.

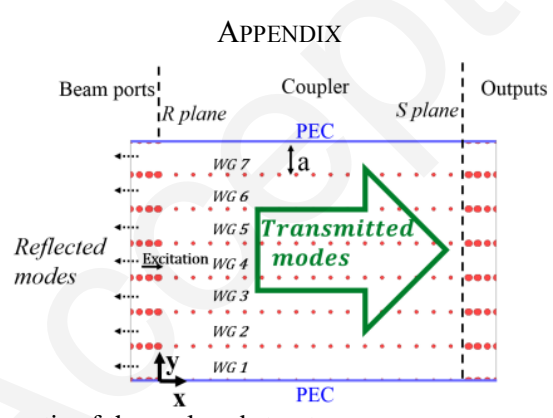

Fig. 12: Schematic of the analyzed structure.

The structure of the waveguide coupler is depicted in Fig. 12. It only comprises 7 input and output waveguides and is surrounded by perfect electric conductor (PEC) boundaries. It is assumed that the coupler is excited by the central waveguide (waveguide 4) and that the power reaching the PEC boundaries is negligible (lower than $-12 \mathrm{~dB}$ ). This means that the length and the width of the coupler allows a complete power transfer between the inputs and outputs.

The S-matrix calculation consists in three steps:

- Derivation of the propagation constants of the transmitted propagating modes within the coupling region.
- Derivation of the amplitude and phase of the transmitted and reflected modes.

- Superposition of the propagating modes in the coupling region.

A. Propagating modes in the coupling region

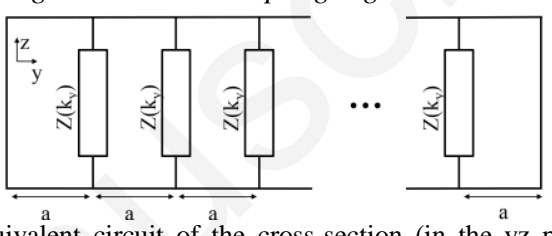

Fig. 13: Equivalent circuit of the cross-section (in the $\mathrm{yz}$ plane) of the coupler region.

The determination of the modes propagating in the coupler region is an extension of the analysis of the transvar coupler proposed in [11]. Only TE modes are considered. The rows of vias are modeled as inductors whose impedance $\mathrm{Z}\left(\mathrm{k}_{\mathrm{y}}\right)$ is provided in [15]. A transverse resonance technique is then enforced to the equivalent circuit in Fig. 13 to derive the propagation constants of the propagating modes along $y$ axis $\left(k_{y}\right)$. It is found that 7 transverse electric modes can propagate within the coupler region. In the following, their amplitude distribution along $y$ is called $C_{m}(y)$, with $m$ an integer number from 1 to 7 .

\section{B. Excitation of the coupler}

The mode amplitudes are obtained by enforcing the continuity of the field along the $\mathrm{R}$ plane. The structure is fed from the central waveguide with a TE10 mode. The transmitted modes travelling within the coupler region can be expressed as

$$
\begin{aligned}
E_{z}^{t} & =\sum_{m=1}^{7} V_{m}^{t} C_{m}(y) e^{-j k_{x m}^{t} x} \\
H_{y}^{t} & =\sum_{m=1}^{7} \frac{V_{m}^{t}}{Z_{m}^{t}} C_{m}(y) e^{-j k_{x m}^{t} x}
\end{aligned}
$$

where $V_{m}^{t}$ is the unknown complex coefficient of the $m^{\text {th }}$ transmitted mode in the coupling region, $k_{x m}^{t}$ is the propagation constant along $x$, and $Z_{m}^{t}$ is the associated wave impedance.

Similarly, the reflected modes travelling backward within the input waveguides can be written as

$$
\begin{aligned}
& E_{z}^{r}=\sum_{g=1}^{7} \sum_{n=1}^{\infty} V_{g n}^{r} \sin \left(\frac{n \pi(y-a(g-1))}{a}\right) \Pi_{g}(y) e^{j k_{x n}^{r} x} \\
& H_{y}^{r}=\sum_{g=1}^{7} \sum_{n=1}^{\infty} \frac{V_{g n}^{r}}{Z_{g n}^{r}} \sin \left(\frac{n \pi(y-a(g-1))}{a}\right) \Pi_{g}(y) e^{j k_{x n}^{r} x}
\end{aligned}
$$

where $V_{g n}^{r}$ is the unknown complex coefficient of the $n^{\text {th }}$ reflected mode in the waveguide $g, k_{x n}^{r}$ is its propagation constant along $\mathrm{x}, Z_{g n}^{r}$ is the 
associated wave impedance and $\Pi_{g}(y)=1$ within the area of waveguide $g$ and is null elsewhere.

As an example, we consider the excitation of the coupler by an incident $\mathrm{TE}_{10}$ mode in guide 4 with a unitary electric field amplitude. The continuity of the fields along the R plane is then expressed by

$$
\begin{aligned}
& \Pi_{4}(y) \sin \left(\frac{\pi(y-3 a)}{a}\right)+E_{z}^{t}=E_{z}^{r} \\
& \frac{\Pi_{4}(y)}{Z_{g n}^{r}} \sin \left(\frac{\pi(y-3 a)}{a}\right)-H_{y}^{t}=H_{y}^{r}
\end{aligned}
$$

where the left-hand side expresses the modes propagating towards positive $\mathrm{x}$ and the right-hand side expresses the modes propagating towards negative $\mathrm{x}$.

By testing (5) with the function $C_{p}(y)$ with $p$ an integer from 1 to 7 , and (6) by $\sin \left(\frac{q \pi(y-a(h-1))}{a}\right) \Pi_{h}$ yields:

$$
\begin{gathered}
\alpha_{4 \mathrm{p} 1}+\sum_{g=1}^{7} \sum_{n=1}^{\infty} V_{g n}^{r} \alpha_{\mathrm{gpn}}=V_{p}^{t} \gamma_{p} \\
\frac{\delta_{h 4}}{2 Z_{4 q}^{r}}-\frac{V_{h q}^{r}}{2 Z_{h q}^{r}}=\sum_{m=1}^{N_{m}} \frac{V_{m}^{t}}{Z_{m}^{t}} \alpha_{\mathrm{hmq}}
\end{gathered}
$$

where

$$
\begin{gathered}
\alpha_{\mathrm{gmn}}=\int_{y=(g-1) a}^{y=g a} \mathrm{C}_{\mathrm{m}}(\mathrm{y}) \sin \left(\frac{n \pi(y-a(g-1))}{a}\right) \mathrm{dy} \\
\gamma_{\mathrm{m}}=\int_{y=0}^{y=7 a} \mathrm{C}_{\mathrm{m}}(\mathrm{y})^{2} \mathrm{dy}
\end{gathered}
$$

and $\delta_{i j}$ is the Kronecker delta.

By using (8) into (7), the amplitude coefficients of the modes travelling in the coupler region $\left[V^{t}\right]$ can be calculated from the following linear system

$$
([D]+[I][\gamma])\left[V^{t}\right]=[G]
$$

where

$$
\begin{gathered}
D_{i j}=\sum_{g=1}^{7} \sum_{n=1}^{\infty} \frac{Z_{g n}^{r} \alpha_{\mathrm{gjn}} \alpha_{\mathrm{gjn}}}{Z_{j}^{t} Z_{n}^{t}} \\
\mathrm{G}_{i}=\alpha_{4 \mathrm{i} 1}+\sum_{n=1}^{\infty} \frac{Z_{g n}^{r} \alpha_{4 \mathrm{in}} Z_{4 n}^{r}}{Z_{41}^{r} Z_{n}^{t}}
\end{gathered}
$$

and $[\mathrm{I}]$ is the identity matrix. The mode amplitudes $V_{g n}^{r}$ can be obtained using (5) or (6).

\section{Superposition of the propagating modes}

The power transmitted to the outputs is computed as a function of the length of the coupler by integrating the field along the cross-section of each output waveguide. The transmitted power between two adjacent waveguides is shown in Fig. 14 and compared to full-wave simulations, with the exact same dimensions as in Section 0. The agreement is excellent, validating the proposed approach.

It is worth mentioning that the reflections occurring at the $S$ plane are neglected since lower than $-35 \mathrm{~dB}$. However, a complete rigorous analysis of the system would be possible by applying the opportune boundary conditions and field continuity along $S$.

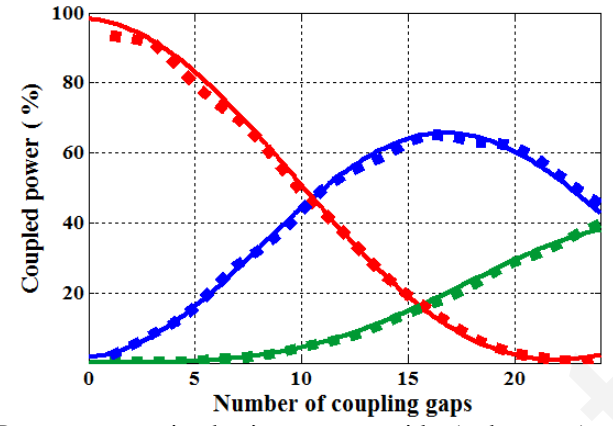

Fig. 14: Power present in the input waveguide (red curves), and power coupled to the first and second adjacent waveguides (blue and green curves, respectively) as functions of the coupler length. Comparison between the results obtained with the presented method (solid curves) and with full-wave simulations (dashed curves).

\section{REFERENCES}

[1] R. C. Hansen, "Phased array antennas," John Wiley \& Sons, Inc., 1998.

[2] W. D. White, "Pattern limitation in multiple-beam antennas," IRE Trans. Antennas and Propag , vol. AP-10, no. 4, pp. 430-436, Jul. 1962.

[3] W. Rotman, "Wide-angle scanning with microwave double-layer pillboxes," IRE Trans. Antennas and Propag, Vol. 6, no.1, pp. 95-105, Jan. 1958.

[4] T. Potelon, M. Ettorre, L. Le Coq, T. Bateman, J. Francey, D. Lelaidier, E. Seguenot, F. Devillers, and R. Sauleau, "A low-profile broadband 32slot continuous transverse stub array for backhaul applications in Eband," IEEE Trans. Antennas and Propag., vol. 65, no. 12, pp. 63076316, Dec. 2017.

[5] J. L. Allen, "A theoretical limitation on the formulation of lossless multiple beams in linear arrays," IEEE Trans. Antennas and Propag, vol. AP-9, no. 7, pp.350-352, Jul. 1961.

[6] S. Stein, "On cross coupling in multiple-beam antennas," IRE Trans. Antennas Propag., Vol. AP-10, No 5, pp. 548-557, Sep. 1962.

[7] K. Tekkouk, M. Ettorre, E. Gandini, and R. Sauleau, "Multibeam pillbox antenna with low sidelobe level and high-beam crossover in SIW technology using the split aperture decoupling method," IEEE Trans. Antennas and Propag , vol. 63, no. 11, pp. 5209-5215, Nov. 2015.

[8] F. Foglia Manzillo, M. Smierzchalski, L. Le Coq, M. Ettorre, J. Aurinsalo, K. T. Kautio, M. S. Lahti, A. E. I. Lamminen, J. Säily, and R. Sauleau, "A wide-angle scanning switched-beam antenna system in LTCC technology with high beam crossing levels for V-band communications," IEEE Trans. Antennas and Propag, vol. 67, no. 1, pp. 541-553, Jan. 2019.

[9] C. Leclerc, M. Romier, H. Aubert, and A. Annabi, "Ka-band multiple feed per beam focal array using interleaved couplers," IEEE Trans. Microw. Theory Tech. , vol. 62, no. 6, pp. 1322-1329, Jun. 2014

[10] M. S. Smith, "Multiple beam crossovers for a lens-fed antenna array," Jour. Inst. of Elec. and Radio Eng., vol. 55, no. 1, pp. 33-36, Jan. 1985.

[11] K. Tomiyasu and S. B. Cohn, "The transvar directional coupler," Proc. of the IRE, vol. 41, no. 7, 1953.

[12] A. Attaran, R. Rashidzadeh, and A. Kouki, "60 GHz low phase error Rotman lens combined with wideband microstrip antenna array using LTCC technology," IEEE Trans. Antennas and Propag., vol. 64, no. 12, pp. 5172-5180, Dec. 2016.

[13] S. G. Hay, S. L. Smith, G. P. Timms, and J. W. Archer, "Three-shapedreflector beam-scanning pillbox antenna suitable for mm wavelengths," IEEE Trans. Antennas and Propag., vol. 59, no. 7, pp. 2495-2501, Jul. 2011.

[14] L.-H. Zhong, Y.-L. Ban, J.-W. Lian, Q.-L. Yang, J. Guo, and Z.-F. Yu, "Miniaturized SIW multibeam antenna array fed by dual-layer $8 \times 8$ Butler matrix," IEEE Antennas and wireless lett., vol. 16, pp. 3018-3021, 2017.

[15] G. G. Macfarlane, "Surface impedance of an infinite parallel-wire grid at oblique angles of incidence," Jour. Inst. Elec Eng., vol. XCII, pt. III A, no. 10 , pp. $1523-1527,1946$ 